\title{
Three-Dimensional Spin Structure on a Two-Dimensional Lattice: $\mathrm{Mn} / \mathrm{Cu}(111)$
}

\author{
Ph. Kurz, G. Bihlmayer, K. Hirai,* and S. Blügel ${ }^{\dagger}$ \\ Institut für Festkörperforschung, Forschungszentrum Jülich, D-52425 Jülich, Germany
}

(Received 17 July 2000)

\begin{abstract}
Based on first-principles vector spin-density total-energy calculations of the magnetic and electronic structure of $\mathrm{Cr}$ and $\mathrm{Mn}$ transition-metal monolayers on the triangular lattice of a (111) oriented $\mathrm{Cu}$ surface, we propose for $\mathrm{Mn}$ a three-dimensional noncollinear spin structure on a two-dimensional triangular lattice as magnetic ground state. This new spin structure is a multiple spin-density wave of three row-wise antiferromagnetic spin states and comes about due to magnetic interactions beyond the nearest neighbors and due to higher order spin interactions (i.e., four spin). The magnetic ground state of $\mathrm{Cr}$ is a coplanar noncollinear periodic $120^{\circ}$ Néel structure.
\end{abstract}

DOI: $10.1103 /$ PhysRevLett.86.1106

In the frontier field of nanomagnetism, understanding the effect of frustration on magnetic properties is one of the current key issues. Exchange bias [1], for example, is a technologically important effect for the magnetic recording industry and the magnetoelectronics, for which frustration plays an important role. In magnetic systems the term frustration refers to the inability to satisfy the competing exchange interactions between neighboring atoms. Frustration is known to be responsible for a number of diverse phenomena such as spin-glass behavior, noncollinear and incommensurate magnetic order, and unusual critical properties. One of the most evident examples of frustration is the so-called geometric frustration of an antiferromagnet on a triangular lattice. In fact, triangular antiferromagnets can be crystallized, e.g., in the form of stacked antiferromagnets. Typical compounds are $\mathrm{RbNiCl}_{3}, \mathrm{VCl}_{2}$, or $\mathrm{CuCrO}_{2}$ [2], and they are localized spin systems. The magnetic properties of these triangular antiferromagnets are almost exclusively described within the framework of model Hamiltonians, the simplest of which is the classical Heisenberg model

$$
H=-\sum_{i, j} J_{i j} \mathbf{S}_{i} \mathbf{S}_{j}
$$

where $J_{i j}$ describes the pairwise (two-spin) exchange interaction between spins at lattice sites $i$ and $j$. $\quad \mathbf{S}$ is the classical spin vector related to the magnetic moment vector $\mathbf{m}$ of localized atomic moments $\mathbf{m}=-g \mu_{B} \mathbf{S}$. Localized spin systems are often well described by restricting the interaction to the antiferromagnetic nearestneighbor (n.n.) one, $J_{i j}=0$ for all $i, j$, except for $J_{(\text {n.n.) }}=$ $J_{1}\left(J_{1}<0\right)$. In this case the minimum energy configuration is the periodic $120^{\circ}$ Néel state in the $(\sqrt{3} \times \sqrt{3}) R 30^{\circ}$ [3] unit cell (cf. Fig. 1b), a two-dimensional noncollinear structure with three atoms per surface unit cell, which consists of coplanar spins forming $\pm 120^{\circ}$ angles between nearest neighbors.

Until now there has been no investigation of twodimensional (2D) itinerant antiferromagnets on a triangular lattice beyond model Hamiltonians. In itinerant
PACS numbers: 75.70.Ak, 71.15.Ap, 75.30.Fv, 75.70.Rf

magnets, the electrons that are responsible for the formation of the magnetic state do participate in the formation of the Fermi surface and hop across the lattice. Thus, it is by no means clear how far a short-ranged n.n. interaction or even how far the Heisenberg model can go in giving a sufficiently good description of the physics of itinerant magnets on a triangular lattice. Monolayers of $\mathrm{Cr}$ and Mn deposited on a substrate with a triangular lattice are ideal candidates for physical realizations of frustrated 2D itinerant antiferromagnets: $\mathrm{Cr}$ and $\mathrm{Mn}$ have been predicted to be 2D antiferromagnets as monolayers on (001) oriented substrates [4]. Triangular lattices are easily provided by (0001) oriented hcp or (111) oriented fcc substrates on which, for example, Mn monolayers [5,6] are grown pseudomorphically or by a pseudohexagonal growth as, for example, of $c(8 \times 2) \mathrm{Mn}$ on $\mathrm{Cu}(100)$ [7].

The aim of this work is to investigate the ground-state spin structure of $\mathrm{Cr}$ and $\mathrm{Mn}$ monolayers on $\mathrm{Cu}(111)$ beyond the (n.n.) Heisenberg model by performing $a b$ initio calculations based on the density-functional theory (DFT) in the local spin-density approximation (LSDA) [8]. This requires the treatment of noncollinear magnetism with a full-potential method (the latter is needed to reliably treat transition-metal surfaces). Only very few calculations of this sort exist today (cf. Refs. [9-11]), and none of them have been applied to two-dimensional systems. We carried out self-consistent calculations with the full-potential linearized augmented plane-wave (FLAPW) method in film geometry [12] as implemented in the program FLEUR. The
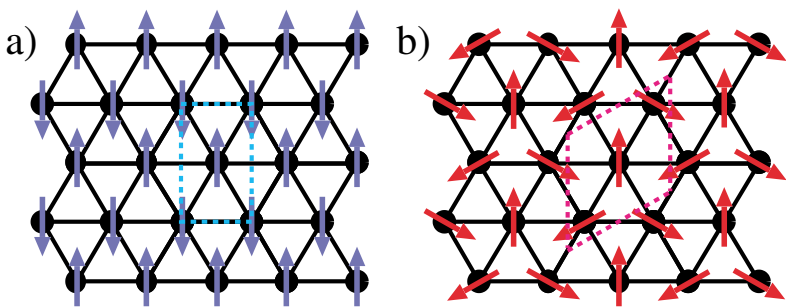

FIG. 1 (color). (a) The row-wise antiferromagnetic structure and (b) the coplanar noncollinear Néel $\left(120^{\circ}\right)$ structure. Indicated are the corresponding two- and three-atom unit cells. 
method has been extended to treat (a) noncollinear magnetism with magnetic moments $M_{i}$ at atom sites $i$ oriented along arbitrarily chosen directions $\hat{\mathbf{e}}_{i}$ as well as (b) incommensurate spiral (or helical) spin-density wave (SSDW) states $\mathbf{M}_{i}=M\left(\cos \mathbf{Q} \mathbf{R}_{i}, \sin \mathbf{Q} \mathbf{R}_{i}, 0\right)$ with wave vectors $\mathbf{Q}$ of the spin-spiral and atom $i$ with the lattice vector $\mathbf{R}_{i}$. With this two-pronged approach we are able to include all spin structures relevant to this work. According to the original formulation of the spin-dependent DFT [13] the magnetization density $\mathbf{m}(\mathbf{r})$ is a vector quantity which we treat similar to Nordström and Singh [10]: The full continuous vector magnetization density $\mathbf{m}(\mathbf{r})$ without any shape approximation is used in the interstitial region between the atoms and in the vacuum region. Around each atom $i$ a (muffin-tin) sphere is defined in which the magnetization density has only one local magnetization axis, $\mathbf{m}_{i}(\mathbf{r})=m_{i}(\mathbf{r}) \hat{\mathbf{e}}_{i}$ [14]. In general [except for some high symmetry magnetic states, e.g., the ferromagnetic state], the magnetization directions $\hat{\mathbf{e}}_{i}$ are not extrema to the LSDA total energy functional $E[n(\mathbf{r}), \mathbf{m}(\mathbf{r})]$. Therefore we work with the constrained DFT as formulated by Dederichs et al. [15] and applied recently by Stocks et al. [16]. The SSDW has been implemented according to Andersson et al. [11] applying the generalized Bloch theorem [17]. More details on the implementation will be published in a forthcoming paper [18].

At first we develop a physical picture by discussing results of unsupported (free standing) monolayers (UML) of $\mathrm{Cr}(111)$ and $\mathrm{Mn}(111)$ taken at the $\mathrm{Cu}$ lattice constant. Since the hybridization between a monolayer and the noble metal substrate is rather small, UML are model systems for magnetic monolayers (ML) on noble metal substrates. Below we will present results including the $\mathrm{Cu}$ substrate. We calculated the total energies $E(\alpha)$ and magnetic moments $M(\alpha)$ constrained along orientations described by an angle $\alpha$ in a two-atom (see inset in upper left panel of Fig. 2) and a $(\sqrt{3} \times \sqrt{3}) R 30^{\circ}$ unit cell with three atoms (see inset in upper right panel of Fig. 2). The angle $\alpha$ was varied in small steps generating a path of quasicontinuously varying orientations connecting high-symmetry magnetic states, i.e., the ferromagnetic (FM) with the row-wise antiferromagnetic (RW-AFM) (Fig. 1a) state, or connecting the FM state with the periodic $120^{\circ}$ Néel state (Fig. 1b) and with a collinear (anti)ferrimagnetic state (FI) at $\alpha=180^{\circ}$ in the corresponding two-atom and three-atom unit cells. The calculations are carried out using 135 or $70 \mathbf{k}_{\|}$-points in the irreducible wedge of the 2D Brillouin zone (2DBZ) of the two-atom and three-atom unit cells, respectively.

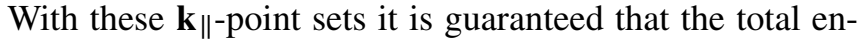
ergies of different unit cells are absolutely converged and directly comparable.

The results are presented in Fig. 2. Consider first Cr: Starting from the RW-AFM solution (upper left panel) and rotating the magnetic moment towards the FM state, the magnetic moment decreases rapidly and finally disappears at $\alpha \approx 60^{\circ}$. Thus, a ferromagnetic solution of the $\operatorname{Cr}(111)$ UML with the $\mathrm{Cu}$ lattice constant does not exist. Al-

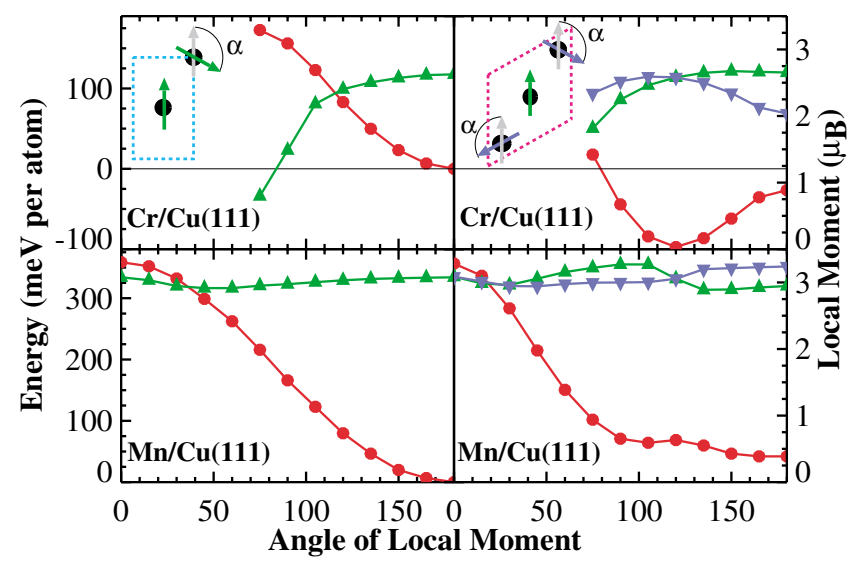

FIG. 2 (color). Calculated total energy per atom (circles) and magnetic moments (triangles) as a function of the rotation angle $\alpha$ of the local moment for the UML of Cr (upper panels) and Mn (lower panels) with the $\mathrm{Cu}(111)$ geometry. The left (right) panels show rotations according to the inset in the upper left (right) panel in the two- (three-) atom unit cell. Generally, the moments of the center atom (up triangles) and the outer atoms (down triangles) differ in the unit cell with three atoms.

though the moment changes drastically, the energy shows a cosinelike behavior in the region where a magnetic solution exists, as the n.n. Heisenberg model predicts for an antiferromagnet. In the three-atom unit cell (upper right panel) the total energy reveals a pronounced minimum at $120^{\circ}$. This minimum and the shape of the energy curve is consistent with the prediction of the Heisenberg model. It is clearly visible that the $120^{\circ}$ configuration is the lowest energy configuration among all configurations studied here. Thus, for $\operatorname{Cr}(111) \mathrm{UML}$ the $120^{\circ} \mathrm{Ne} e l$ state is the magnetic ground state predicted by the present investigation.

Now turning to $\mathrm{Mn}$ and comparing the results in the twoatom unit-cell (lower left panel) with those of $\mathrm{Cr}$ (upper left panel) we find the behavior of $\mathrm{Mn}$ and $\mathrm{Cr}$ is very similar, i.e., the energy curve is cosinelike and $\mathrm{Mn}$ prefers to be RW-AFM. However, in contrast to $\mathrm{Cr}$ the ferromagnetic state exists, and the magnetic moments change only within a narrow range, $2.9 \mu_{B}-3.05 \mu_{B}$, as a function of the relative spin orientation. The Mn system with 3 atoms per unit cell (lower right panel) reveals, however, two surprises: (i) The RW-AFM state is lower in energy than the $120^{\circ}$ Néel state and any other magnetic state investigated so far. (ii) The total energy of the Mn system does not exhibit a minimum at $120^{\circ}$, as predicted by the n.n. Heisenberg model, but a local maximum.

Obviously, these surprises cannot be explained within the n.n. Heisenberg model. Extending it to exchange interactions between the first, second, and third nearest neighbors, $J_{1}, J_{2}$, and $J_{3}$, respectively, the energy per atom as a function of the angle $\alpha$ is given by $E_{2 \mathrm{AT}}^{2 \text {-spin }}(\alpha)=-2\left(J_{1}+\right.$ $\left.J_{2}+3 J_{3}\right)-4\left(J_{1}+J_{2}\right) \cos \alpha$ and $E_{3 \mathrm{AT}}^{2 \text {-spin }}(\alpha)=-6 J_{2}-$ $2\left(J_{1}+J_{3}\right)(2 \cos \alpha+\cos 2 \alpha)$ for the two-atom and threeatom unit cell, respectively. Clearly, the magnetic ground state depends on the sign and magnitude of the different 
$J$ 's. A zero-temperature phase-diagram calculated in the $\left(J_{1}, J_{2}, J_{3}\right)$ space reveals the possibility of four different magnetic ground states: FM, RW-AFM, $120^{\circ}$ Néel, and SSDW, all specified by a SSDW with a single wave vector $\mathbf{Q}_{\|}$either at one of the high-symmetry points or at the high-symmetry lines $\bar{\Gamma}-\overline{\mathrm{K}}-\overline{\mathrm{M}}-\bar{\Gamma}$ in the $2 \mathrm{DBZ}$ (see inset of Fig. 3). The high-symmetry points $\bar{\Gamma}, \overline{\mathrm{K}}$, and $\overline{\mathrm{M}}$ correspond to the FM, $120^{\circ}$ Néel, and RW-AFM state, respectively. For transition metals it is known (see, e.g., Ref. [19]) that the sign and magnitude of the exchange interactions $J$ are rapidly varying functions of the $d$-band filling. Thus, incommensurate SSDWs as possible magnetic ground states cannot a priori be excluded.

We have calculated the total energy $E\left(\mathbf{Q}_{\|}\right)$and the magnetic moments $M\left(\mathbf{Q}_{\|}\right)$for a discrete set of $\mathbf{Q}_{\|}$vectors along the high-symmetry lines (Fig. 3). The calculations are carried out with one atom per unit cell and

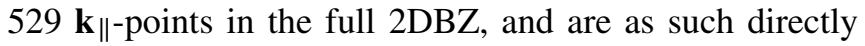
comparable to the results of the two- and three-atom unit cells. We found the following: Among all the SSDWs calculated, the high-symmetry points have the lowest

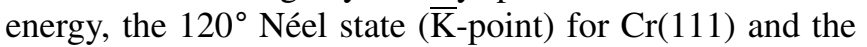
RW-AFM state ( $\overline{\mathrm{M}}$-point) for $\mathrm{Mn}(111)$. For $\mathrm{Mn}, M\left(\mathbf{Q}_{\|}\right)$is nearly a constant but the $\mathrm{Cr}$ moments change drastically, as no stable ferromagnetic solution could be found for $\mathrm{Cr}(111)$. One more interesting observation is the local minimum of $E\left(\mathbf{Q}_{\|}\right)$for $\mathrm{Mn}$ on the line $\bar{\Gamma}-\overline{\mathrm{K}}$, which is only $21 \mathrm{meV}$ higher in energy than the RW-AFM state. We expect that a small change in the $d$-band filling, e.g., due to alloying with $\mathrm{Fe}$, may change the energetics, and an incommensurate SSDW as magnetic ground state will occur.

A second important point to be noticed is that for Mn the energy dependence $E_{3 \mathrm{AT}}(\alpha)$ of Fig. 2 is not in agreement

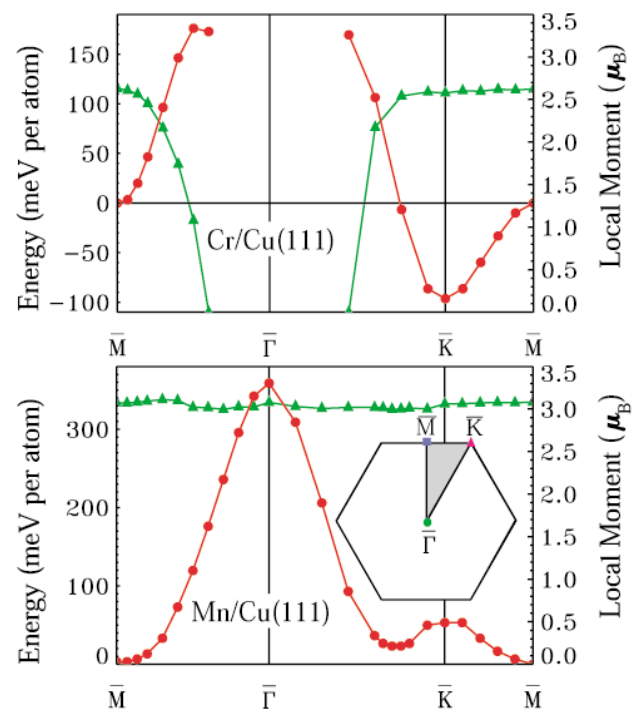

FIG. 3 (color). Calculated total energies (circles) and magnetic moments (triangles) for spin-spiral states as a function of the 2D wave vector $Q_{\|}$along the high symmetry lines of the 2DBZ (see inset) for $\mathrm{Cr}$ (top panel) and $\mathrm{Mn}$ (bottom panel) at $\mathrm{Cu}(111)$. with the functional form of $E_{3 \mathrm{AT}}^{2 \text {-spin }}(\alpha)$ for $\alpha$ around $120^{\circ}$. From $E_{3 \mathrm{AT}}^{2 \text {-spin }}(\alpha)$ and $J_{1}+J_{3}<0$, which describes well the overall behavior of $E_{3 \mathrm{AT}}(\alpha)$, a minimum at $\alpha=120^{\circ}$ is expected, but a maximum was found. Because of this maximum at $120^{\circ}$, a term $E(\alpha) \sim \cos 3 \alpha$ seems to be missing to match the results, a term which is not produced by the Heisenberg model for any $J_{i j}$ [20]. This is indicative of higher order exchange interactions, i.e., exchange interactions of more than two spins. Motivated by the perturbative treatment [21] of the Hubbard model in fourth order perturbation theory in the case of strong Coulomb interaction, the $a b$ initio results suggest the four-spin interaction as lowest order correction to the Heisenberg model. The classical spin Hamiltonian of the four-spin interaction is written as

$$
\begin{gathered}
H_{4 \text {-spin }}=-\sum_{i j k l} K_{i j k l}\left[\left(\mathbf{S}_{i} \mathbf{S}_{j}\right)\left(\mathbf{S}_{k} \mathbf{S}_{l}\right)+\left(\mathbf{S}_{j} \mathbf{S}_{k}\right)\left(\mathbf{S}_{l} \mathbf{S}_{i}\right)\right. \\
\left.-\left(\mathbf{S}_{i} \mathbf{S}_{k}\right)\left(\mathbf{S}_{j} \mathbf{S}_{l}\right)\right] .
\end{gathered}
$$

Within the n.n. approximation $\left(K_{i j k l}=K_{1}\right.$ for all minimum distance diamond clusters with four sites, $K_{i j k l}=0$ otherwise), the energy per atom of the four-spin interaction in the three-atom unit cell, $E_{3 \mathrm{AT}}^{4 \text {-spin }}$, has a functional form, $E_{3 \mathrm{AT}}^{4 \text {-spin }}(\alpha)=-K_{1}(4+8 \cos 3 \alpha)$, consistent with result $E_{3 \mathrm{AT}}(\alpha)$ if $K_{1}<0$, which explains surprise (ii).

For Mn, the lowest energy magnetic state found so far is the RW-AFM state, which corresponds to the commensurate SSDW state with one single $\mathbf{Q}_{\|}$vector at the $\bar{M}$ point of the 2DBZ, and the RW-AFM is also called single- $\mathbf{Q}_{\|}$ (1Q) state. In the $2 \mathrm{DBZ}$ there are three $\overline{\mathrm{M}}$ points which are equivalent in symmetry, but are different to each other with $\mathbf{Q}_{\|}$vectors, $\mathbf{Q}^{(k)}$, for $k=1,2,3$. Within the Heisenberg model the energy of each SSDW denoted by one of the three wave vectors $\mathbf{Q}^{(k)}$ or any SSDW being an orthogonalized linear combination of those are degenerate. The four-spin interaction lifts this degeneracy and multiple SSDWs [22] may become lower in energy. For Mn we predict the existence of a new magnetic ground state, a so-called triple- $\mathbf{Q}_{\|}(3 Q)$ state, $15 \mathrm{meV} /$ atom lower in energy than the $1 Q$ state. The $3 Q$ state is a three-dimensional noncollinear spin structure (see Fig. 4) with four

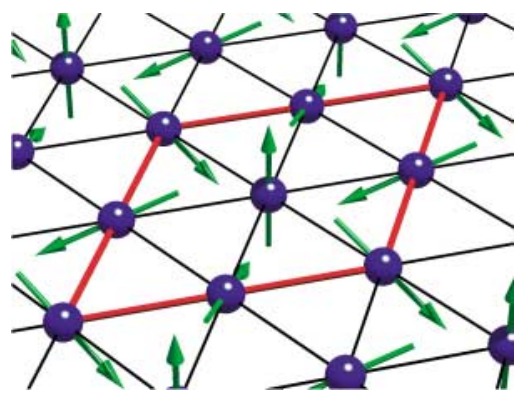

FIG. 4 (color). An image of the magnetic $3 Q$ structure, with spins pointing in all three directions of the spin space. Note that, due to the neglect of the spin-orbit interaction, only the relative orientiation of the moments is specified. 
TABLE I. Energy differences relative to the RW-AFM (1Q) state in $\mathrm{meV} /$ atom for different magnetic configurations.

\begin{tabular}{ccccrc}
\hline \hline UML & FM & FI & SSDW $@ \bar{\Gamma}-\overline{\mathrm{K}}$ & Néel & $3 Q$ \\
\hline $\mathrm{Cr} / \mathrm{Cu}$ & No & -26 & No & -97 & -6 \\
$\mathrm{Mn} / \mathrm{Cu}$ & 358 & 44 & $21 @ 0.7 \overline{\mathrm{K}}$ & 68 & -15 \\
$\mathrm{ML}$ & FM & FI & SSDW $@ \bar{\Gamma}-\overline{\mathrm{K}}$ & Néel & $3 Q$ \\
$\mathrm{Cr} / \mathrm{Cu}$ & $\mathrm{No}$ & $\ldots$ & No & -53 & $\approx 1.5$ \\
$\mathrm{Mn} / \mathrm{Cu}$ & 261 & $\cdots$ & $31 @ 0.6 \overline{\mathrm{K}}$ & 77 & -17 \\
\hline \hline
\end{tabular}

chemically identical atoms per surface unit cell, and is formed as a linear combination of the three RW-AFM $(1 Q)$ structures orthogonal in spin space, each having one of the three $\mathbf{Q}_{\|}^{(k)}$ vectors of the $\bar{M}$ points:

$$
\mathbf{m}\left(\mathbf{r}+\mathbf{R}_{i}\right)=m(\mathbf{r}) \times \frac{1}{\sqrt{3}} \sum_{k=1}^{3} e^{i \mathbf{Q}_{\|}^{(k)} \mathbf{R}_{i}} \hat{\mathbf{e}}^{(k)},
$$

where the $\hat{\mathbf{e}}^{(k)}$ are orthogonal unit vectors in spin space. The energy difference $\Delta E=E_{3 Q}-E_{1 Q}$ has been obtained by $a b$ initio calculations with four atoms per unit

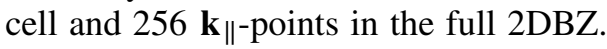

The calculations have been repeated including a $\mathrm{Cu}(111)$ substrate modeled by four layers of $\mathrm{Cu}$. Thus, the number of atoms included in the calculation is $5 \times$ as large as for the UML above and amounts for the $3 Q$ state to calculations with 20 atoms per unit cell carried out with

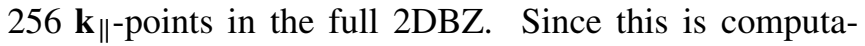
tionally demanding we repeated the calculations only for the high-symmetry states FM, $120^{\circ}$ Néel, RW-AFM (1Q), $3 Q$, and the SSDW state around the local minimum at the high-symmetry line $\bar{\Gamma}-\bar{K}$. The interlayer distances between $\mathrm{Cr}$ or $\mathrm{Mn}$ and the $\mathrm{Cu}$ layers have been optimized by force calculations using seven layer thick $\mathrm{Cu}$ substrate. The results (ML) are summarized in Table I and compared to the results without substrate (UML). We find the fact that the substrate changes the energy differences between the different magnetic states, but does not alter the physical picture developed above.

In summary, we investigated the magnetic structure of the itinerant antiferromagnets $\mathrm{Cr}$ and $\mathrm{Mn}$ on the triangular lattice of the $\mathrm{Cu}(111)$ substrate. Treating noncollinear magnetism in real and reciprocal space we covered a complete set of relevant spin structures. We predict a three-dimensional noncollinear spin structure on a twodimensional triangular lattice as magnetic ground state for $\mathrm{Mn}$ and the two-dimensional noncollinear $120^{\circ}$ Néel state for $\mathrm{Cr}$. While the numerical results come from $a b$ initio calculations, the interpretation of these data is performed with the help of a model Hamilitonian. We believe that the $3 Q$ structure can be experimentally realized since the spin-orbit interaction, which is typically 2 orders of magnitude smaller than the exchange interaction and neglected in this work, will stabilize the long-range order at finite temperature, but will leave the $3 Q$ structure basically unchanged.

Two of us (P. K. and S. B.) thank Lars Nordström and Leonid Sandratskii for many fruitful discussions. Work was supported by the DFG under Grant No. BL444/1-1 and the TMR Networks, Contracts No. EMRX-CT96-0089 and No. FMRX-CT98-0178.

*Permanent address: Department of Physics, Nara Medical University, Kashihara, Nara 634-8521, Japan.

†Electronic address: s.bluegel@fz-juelich.de

[1] For a recent review, see J. Nogues and I. K. Schuller, J. Magn. Magn. Mater. 192, 203 (1999).

[2] For recent reviews, see H. Kawamura, J. Phys. Condens. Matter 10, 4707 (1998); M. F. Collins and O. A. Petrenko, Can. J. Phys. 75, 605 (1997).

[3] $R 30^{\circ}$ means a rotation of the surface supercell by $30^{\circ}$ with respect to the elementary surface unit-cell.

[4] S. Blügel, M. Weinert, and P. H. Dederichs, Phys. Rev. Lett. 60, 1077 (1988); S. Blügel, D. Drittler, R. Zeller, and P. H. Dederichs, Appl. Phys. A 49, 547 (1989).

[5] A. S. Arrot, B. Heinrich, S. T. Purcell, J. F. Cochran, and K. B. Urquhart, J. Appl. Phys. 61, 3721 (1987).

[6] W. L. O'Brien and B. P. Tonner, J. Vac. Sci. Technol. A 13, 1544 (1995).

[7] T. Flores, M. Hansen, and M. Wuttig, Surf. Sci. 279, 251 (1992).

[8] V.L. Moruzzi, J. F. Janak, and A. R. Williams, Calculated Electronic Properties of Metals (Pergamon, New York, 1978).

[9] L. M. Sandratskii, Adv. Phys. 47, 91 (1998).

[10] L. Nordström and D. J. Singh, Phys. Rev. Lett. 76, 4420 (1996).

[11] P. H. Andersson, L. Nordström, and O. Eriksson, Phys. Rev. B 60, 6765 (1999).

[12] E. Wimmer, H. Krakauer, M. Weinert, and A. J. Freeman, Phys. Rev. B 24, 864 (1981); M. Weinert, E. Wimmer, and A. J. Freeman, Phys. Rev. B 26, 4571 (1982).

[13] U. von Barth and L. Hedin, J. Phys. C 5, 1629 (1972).

[14] This is an excellent approximation for $3 d$ metals.

[15] P. H. Dederichs, S. Blügel, R. Zeller, and H. Akai, Phys. Rev. Lett. 53, 2512 (1984).

[16] G. M. Stocks et al., Philos. Mag. B 78, 665 (1998).

[17] C. Herring, Magnetism IV, edited by G. Rado and H. Suhl (Academic Press, New York, 1966), Chaps. V and XIII.

[18] Ph. Kurz, G. Bihlmayer, and S. Blügel (to be published).

[19] K. Terakura, N. Hamada, T. Oguchi, and T. Asada, J. Phys. F 12, 1661 (1982).

[20] This is strictly true only if $M(\alpha)=$ const. For Mn this condition is fulfilled to a very good approximation.

[21] M. Takahashi, J. Phys. C 10, 1289 (1977).

[22] For a triangular lattice there exists a competition between a $1 Q$ and a $3 Q$ state, which is determined by the sign of $K_{1}$, as the energy difference $\Delta E=E_{3 Q}-E_{1 Q}=32 / 3 K_{1}$. The possibility of a double- $Q_{\|}$state can be discarded based on an analogy of fcc antiferromagnets: T. Jo and K. Hirai, J. Phys. Soc. Jpn. 53, 3183 (1984). 